Method From January 2011 through September 2015, A retrospective study was performed in 27 patients who had hyperacute ischemic stroke and were treated by primary intracranial stenting for refractory re-occlusion during stent-based mechanical thrombectomy. We studied radiologic appearance, clinical presentation and follow up outcomes.

Result Of the 27 patients, 11 patients (40\%) showed radiologic and clinical improvement, 5 patients (18\%) showed clinical improvement with in-stent stenosis, 6 patients $(22 \%)$ had complication with hemorrhagic transformation and 4 patients (14\%) failed recanalization or severe stenosis. In all cases, we use Solitaire FR stent. 6 patients (22\%) were TICI grade III, 10 patients $(37 \%)$ were TICI grade IIb, 4 patients $(14 \%)$ were TICI grade IIa, and 4 patients (14\%) were TICI grade I. After 3 months, 15 patients (55\%) were below mRS 3 points and after 6 month, 18 patients (67\%) were below mRS 3 points.

Conclusions These results indicate that primary intracranial stenting for refractory re-occlusion during stent-based mechanical thrombectomy treatment in acute ischemic stroke is effective technique. Further studies are needed to evaluate longterm occlusion and in-stent stenosis.

Disclosures S. Sheen: None. J. Shin: None.

\section{E-025 FIRST REPORTED CASE OF MECHANICAL THROMBECTOMY FOR ACUTE ISCHEMIC STROKE IN AN INDIVIDUAL WITH A TOTAL ARTIFICIAL HEART}

${ }^{1} \mathrm{M}$ Nezhad, ${ }^{2} \mathrm{P}$ Eboli, ${ }^{3} \mathrm{M}$ Austin, ${ }^{1} \mathrm{~K}$ Schlick, ${ }^{2} \mathrm{M}$ Alexander. ${ }^{1}$ Neurology, Cedars-Sinai Medical Center, Los Angeles, CA; ${ }^{2}$ Neurosurgery, Cedars-Sinai Medical Center, Los Angeles, CA; ${ }^{3}$ Radiology, Cedars-Sinai Medical Center, Los Angeles, CA

\subsection{6/neurintsurg-2016-012589.97}

Background With the results of recent thrombectomy trials, endovascular therapy (EVT) is standard of care in appropriately selected patients with acute ischemic stroke (AIS). We report the first case of mechanical thrombectomy for a patient supported by a total artificial heart (TAH) with AIS.

Clinical Presentation A 37 year old male patient with ischemic cardiomyopathy was found to have right gaze preference, left facial droop, and global aphasia 9 days following implantation of a TAH. Prior to ictus, antithrombotic regimen included daily aspirin and heparin infusion at therapeutic levels. Acute imaging revealed a $6 \mathrm{~mm}$ left M2 thrombus with associated perfusion mismatch seen in the left MCA territory along with a smaller matched perfusion defect seen in the right MCA territory.

Intervention Following confirmation of lesion amenable to mechanical thrombectomy, patient underwent successful TICI 3 revascularization of the left MCA territory with a Solitaire stent retriever. Repeat perfusion imaging revealed resolved left MCA territory mismatch with persistent right MCA matched defect. His speech improved to an expressive aphasia following revascularization.

Conclusion EVT has been proven to be safe and efficacious for AIS. Prior reports have demonstrated benefit for patients with concurrent mechanical circulatory support devices; however, our report is the first case demonstrating efficacy with a $\mathrm{TAH}$ device. Presence of mechanical circulatory support devices should not exclude EVT in individuals with AIS.

Disclosures M. Nezhad: None. P. Eboli: None. M. Austin: None. K. Schlick: None. M. Alexander: None.

\section{E-026 DIAGNOSIS AND MANAGEMENT OF THORACIC AND SHOULDER ARTERIOVENOUS MALFORMATIONS}

W Yakes. Vascular Malformation Center, Englewood, CO

\subsection{6/neurintsurg-2016-012589.98}

Purpose To determine the efficacy of Endovascular Repair of Thoracic and Shoulder Arteriovenous Malformations (AVMs). Previous reports have documented the futility of nBCA and amputation in treating these lesions in this specific anatomy. Materials and methods Twelve patients ( 8 female, 4 male) presented for repair of shoulder and thoracic AVMs. Three patients had extension of AVM to the supraclavicular and axillary areas. Two patients had multiple AVMs. Seven patients had previous failed therapies (embo: PVA/coils/gelfoam; surgeries: excisions/arterial bypass). All patients underwent ethanol endovascular AVM repair; four patients had additional coil embolizations (132 treatments). Patient age range 18-76 years; mean age 36 .

Results Eleven patients are cured at long-term arteriographic follow-up (follow-up 22-192 months; mean follow-up: 42 months). One patient with bilateral shoulder AVM and multiple other AVMs therapy is on-going. Complications include two patients with minor superficial blisters, one patient with transient left radial nerve injury with complete recovery and one patient with clot embolus to hand, $\mathrm{Rx}$ with urokinase w/ distal $3^{\text {rd }}$ phalanx removed. Thus, major complications were 2/132 procedures, one being transient.

Conclusions A report of shoulder AVM repair in JVIR documented failure of nBCA approach even coupled with quadrant amputation whereby recurrence was universal. These authors stated that shoulder AVMs were not possible to treat. This report documents that cure of these difficult lesions is possible with ethanol endovascular approaches and direct puncture approaches. No other publications in world literature documents cure of AVMs in this anatomy.

Long-term cures are noted with the use of ethanol, and ethanol and coils to successfully treat these complex, problematic lesions. A low major complication rate is noted. This patient series finally documents a curative procedure for this daunting lesion.

Disclosures W. Yakes: None.

\section{E-027 MECHANICAL THROMBECTOMY FOR ACUTE ISCHEMIC STROKE IN POST-SURGICAL PATIENTS}

${ }^{1}$ M Litao, ${ }^{1} \mathrm{H}$ Bhamra, ${ }^{2} \mathrm{~K}$ DeSousa, ${ }^{2} \mathrm{E}$ Raz, ${ }^{3} \mathrm{E}$ Nossek, ${ }^{1} \mathrm{~A}$ Favate, ${ }^{4} \mathrm{M}$ Shapiro, ${ }^{5} \mathrm{~T}$ Becske, ${ }^{2} \mathrm{P}$ Nelson. 'Neurology, New York University, New York, NY; ${ }^{2}$ Radiology, New York University, New York, NY; ${ }^{3}$ Neurosurgery, Maimonides Medical Center, New York, NY; ${ }^{4}$ Neurology/Radiology, New York University, New York, NY; ${ }^{5}$ Neurology, Rochester Regional Health, Rochester, NY

\subsection{6/neurintsurg-2016-012589.99}

Introduction Ischemic strokes in the post-surgical period may negatively impact clinical outcome. Major surgery within the previous 14 days is a relative contraindication to intravenoustPA administration. Mechanical thrombectomy may thus become a valid treatment option. We present our experience in a series of patients who presented with a clinical stroke syndrome in the post-surgical period who underwent emergent angiography with intent to treat.

Methods This is a retrospective review of patients who were treated from January 2012 to March 2015 in our institution. 